EESTI NSV TEADUSTE AKADEEMIA TOIMETISED. XI KOIDE FOOSIKALIS-MATEMAATILISTE JA TEHNILISTE TEADUSTE SEERIA. 1962, NR. 1

ИЗВЕСТИЯ АКАДЕМИИ НАУК ЭСТОНСКОЙ ССР. ТОМ ХІ СЕРИЯ ФИЗИКО-МАТЕМАТИЧЕСКИХ И ТЕХНИЧЕСКИХ НАУК. 1962, Ni 1

\title{
РАЗДЕЛЕНИЕ ПРИРОСТА ПРОИЗВЕДЕНИЯ
}

\author{
А. ХУМАЛ, \\ академик Академии наук Эстонской ССР
}

\section{1. О способах разделения}

При решении некоторых задач экономической статистики, а также в отдельных отраслях техники возникает вопрос, как разделить изменение произведения нескольких величин между переменными сомножителями, с тем чтобы количественно установить влияние каждого из них. К решению этого вопроса естественно приступить с рассмотрения простейшего случая: если в произведении двух величин одну из них оставить без изменения и другую увеличить или уменьшить на несколько процентов, то изменение произведения составляет столько же процентов в том же направлении. Здесь. конечно, весь прирост произведения, будь он положителен или оfрицателен, следует отнести на счет переменного сомножителя.

В случае же одновременного изменения обоих сомножителей произведение изменяется под влиянием каждого из них. Например, увеличение одного сомножителя на $20 \%$ и другого на $44 \%$ влечет за собой увеличение их произведения на $72,8 \%$, так как $1,2 \cdot 1,44=1,728$ и вообще $(1+\alpha)(1+\beta)=1+\alpha+\beta+\alpha \beta$. Находящиеся в употреблении способы разделения прироста произведения приводят к различным результатам, хотя все они исходят из того обстоятельства, что увеличению только одного из двух сомножителей (на $20 \%$ или на $44 \%$ ) соответствовало бы точно такое же увеличение произведения, и поэтому выделяют из $72,8 \%$ сперва $20 \%$ и $44 \%$, выражающие влияние отдельно взятых сомножителей, чтобы затем перейти к разделению остающихся $8,8 \%$. Способы расходятся только в разделения этих оставшихся $8,8 \%$. Наиболее известны следующие три разделения члена $\alpha \beta$ между сомножителями:

1) на $\frac{\alpha \beta}{2}$ и $\frac{\alpha \beta}{2}$, т. е. на равные части

2) на $\frac{\alpha}{\alpha+\beta} \alpha \beta$ и $\frac{\beta}{\alpha+\beta} \alpha \beta$, т. е. на части, пропорциональные приростам сомножителей

3) на $\frac{a^{2}}{2}$ и $\alpha\left(\beta-\frac{a}{2}\right)$ при $\alpha \leqslant \beta$.

В случае $\alpha=0,2$ и $\beta=0,44$ эти части, соответственно, таковы: 1) $4,4 \%$ и $4,4 \%$; 2) $2,75 \%$ и $6,05 \%$; 3) $2 \%$ и $6,8 \%$. Так, результатами разделения всего прироста произведения будут по первому способу $24,4 \%$ и $48,4 \%$, по второму $22,75 \%$ и $50,05 \%$ и по третьему $22 \%$ и $50,8 \%$ (от первоначального значения произведения).

Нетрудно обнаружить, однако, что ни один из этих вариантов не может быть признан правильным. Поскольку $1,44=1,2 \cdot 1,2$, то произведение 1,728 можно и рассматривать как $1,2^{3}$, т. е. как произведение трех равных сомножителей. Другими словами, если в произведении трех величин каждую из них увеличить на $20 \%$, то оно увеличится на $72,8 \%$. Естественно считать влияния таких сомножителей равными, так 
что $72,8 \%$ или $728 \%$ следует разделить на три равные части, по $242 \frac{2}{3} \%$ каждая. Естественно также считать в случае, когда произведение нескольких величин увеличивается на $72,8 \%$ и одна из них на $20 \%$, что соответствующая ее изменению часть прироста произеедения есть $242 \frac{2}{3} \%$ (от его начального значения); при этом безраз. лично, содержит ли произведение еще два переменных сомножителя (или три, или более трех) или только один, так как в произведении всегда можно произвольно сгруппировать сомножители и любую группу сомножителей заменить их произведением.

Следовательно, при увеличении одного из сомножителей на $20 \%$ и другого на $44 \%$ прирост произведения, составляющий $72,8 \%$ от начального значения, распадается на $242 \frac{2}{3} \%$ и $485 \frac{1}{3} \%$ (того же значения) и эти части выражают влияние изменения сомножителей на произведение.

\section{2. Нормальное разделение}

В соответствин с изложенными принципами решается задача и при общих данных. Пусть величина $p$ превращается в $\lambda p$ и величина $q$ в $\mu q$. Их произведение $p q$ имеет тогда прирост $(\lambda \mu-1) p q$. Если найти $n$ так, что $\mu=\lambda^{n}$, то части прироста произведения, соответствующие влиянию изменения сомножителей, будут $\frac{1}{1+n}(\lambda \mu-1) p q$ и $\frac{n}{1+n}(\lambda \mu-1) p q$. Поскольку из уравнения $\mu=\lambda^{n}$ следует $n=\log _{\lambda} \mu=\frac{\log \mu}{\log \lambda}$, то $1+n=\frac{\log \lambda+\log \mu}{\log \lambda}$, так что прирост произведения разделяется на части $\frac{\log \lambda}{\log \lambda+\log \mu}(\lambda \mu-1) p q$ и $\frac{\log \mu}{\log \lambda+\log \mu}(\lambda \mu-1) p q$.

Такое разделение прироста произведения может быть названо нормальным. Название оправдывается тем, что полученное правило разделения остается в силе при любом числе сомножителей, а именно: прирост произведения разделяется между переменными сомножителями пропорционально логарифмам их коэффициентов изменения. Действительно, в случае любого числа сомножителей можно какой-нибудь один из них обозначить через $p$ и его коэффициент изменения через $\lambda$, а само пронзведение через $T$ и его коэффициент изменения через $\Psi$; тогда влиянию изменения этого сомножителя соответствует часть прироста произведения, равная $\frac{\log \lambda}{\log \Psi}(\Psi-1) T$. При этом Безразлично, сколько переменных сомножителей содержит произведение и каковы их коэффициенты изменения $\lambda, \mu, \ldots, \omega$, так как $\lambda \mu \ldots \omega=\Psi$ и поэтому $\frac{\log \lambda}{\log \Psi}+\frac{\log \mu}{\log \Psi}+\ldots+\frac{\log \omega}{\log \Psi}=1$.

\section{3. Значимость хода изменения сомножителей}

Нормальное разделение прироста произведения столь явно представляется правильным и универсальным, что применение других способов разделения кажется просто ошибочным. Но это только на первый взгляд; в действительности же нормальное разделение прироста является лишь одним из многообразных возможных способов его разделеняя. Дело в том, что во всех случаях, когда сомножители изменяются во времени (или вообще по одному и тому же параметру) вполне определенным и точно известным образом, можно с полной достоверностью установить действительно правильное разделение прироста их произведения между ними. Например, если известно, что произведение двух величин увеличивается на $72,8 \%$ таким образом, что эти величины изменяются попеременно в следующие четыре этапа: 
1) первая из них увеличивается на $10 \%$, а вторая сохраняет свое начальное значение,

2) первая сохраняет достигнутый уровень, а вторая увеличивается на $30 \%$,

3) первая увеличивается еще на $10 \%$ (от своего первоначального значения), а вторая остается на прежнем уровне,

4) -первая опять постоянна, а вторая увеличивается на дальнейшие 14\% (or своего первоначального значения),

то задача решается довольно просто. Весь прирост произведения теперь складывается из четырех частей: $10 \%, 33 \%, 13 \%$ и 16,8\% (от первоначального значения произведения), причем первая и третья явно обусловлены измененнями перзого сомножителя, а вторая и четвертая - второго. Следовательно, при таком ходе изменения сомножителей прирост их произведения распадается на $23 \%$ и $49,8 \%$.

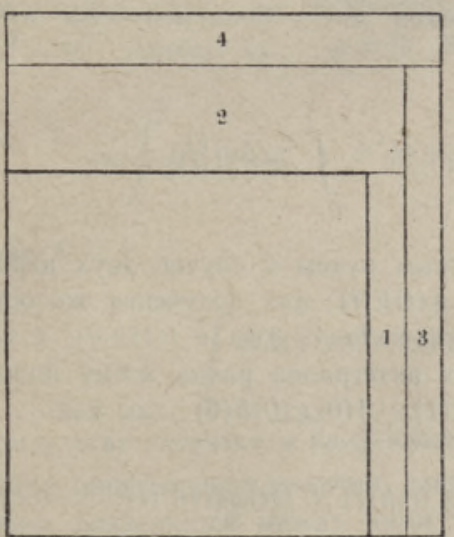

Фиг. 1.

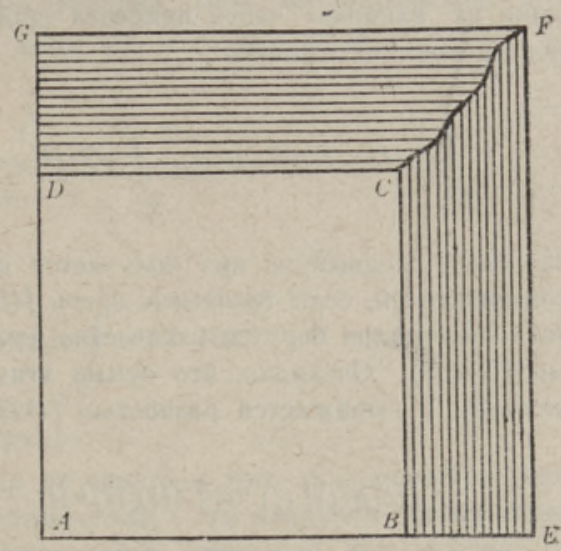

Фиг. 2.

На фиг, 1 начальное значение произведения представлено в виде площади квадрата, стороны которого равны единице. Увеличение первого сомножителя озпачает удлинение горнзонтальной стороны, увеличение второго - удлинение вертикальной стороны. Части прироста произведения, соответствуюшие приведенным выше.четырем этапам, представлены площадями прибавляюшихся прямоугольников, обозначенных номерами $1,2,3$ и 4 .

Рассмотренный ход изменсния сомножителей, хотя он вполне возможен в действнтельности, здесь сконструирован только как введение в обсуждение более общего случая. Пусть произведение двух переменных величин (или произведение их коэффищиентов изменения) изображается площадью квадрата ( $A B C D$ на фиг. 2), превра щающегося в прямоугольник $A E F G$, причем в ходе изменения сомножителей верхняя правая вершина переходит из точки $C$ по некоторой линии в точку $F$. Поскольку эту линию можно составить (мысленно с любой желаемой точностью) из сколь угодно малых горизонтальных и вертикальных элементов попеременно, то она разделяет изображающую прирост произведения площадь $B E F G D C$ на две части: $C B E F$ и $F G D C$, представляющие, соответственно, влияние изменения первого и второго со миожителей.

Если взять декартову систему координат так, что $A, B$ и $D$ означают точки $(0 ; 0),(1 ; 0)$ и $(0 ; 1)$, а $E$ и $G$ - точки $(\lambda ; 0)$ и $(0 ; \mu)$, то линия $C F$ может быть дана своими параметрическими уравнениями $x=f(t)$ и $y=g(t)$. Пусть при этом параметр $t$ подобран так, что $t=0$ в точке $C$ и $t=1$ в точке $F$, т. е. $f(0)=1=g(0), f(1)=\lambda$ и $g(1)=\mu$. (Если $t$ означает время, то изменения величин рассматриваются в промежутке времени $0 \leqslant t \leqslant 1$ ) Части прироста произведе- 
ния, выражающие влияние изменения сомножителей, т. е. площади $C B E F$ и $F G D C$, определены тогда, как известно, в виде интегралов

$$
\int_{0}^{1} f^{\prime}(t) g(t) d t \quad \text { и } \int_{0}^{1} g^{\prime}(t) f(t) d t
$$

причем ввиду того, что их сумма явно равна разности $f(1) g(1)-f(0) g(0)$, или просто $\lambda \mu-1$, достаточно найти только один из них путем интегрирования. Таково общее, принциниально строгое решение задачи в случае двух переменных сомно жителей,

Следует отметить, что таким же образом решается задача и в случае любого числа переменных сомножителей. Если их, например, три и все они даны в виде функций одного и того же параметра $t$ и обозначены $f(t), g(t)$ и $h(t)$, то соответствующие их влиянию части прироста пронзведения могут быть получены в виде следующих трех интегралов:

$$
\int_{0}^{1} f^{\prime}(t) g(t) h(t) d t . \quad \int_{0}^{1} g^{\prime}(t) f(t) h(t) d t \quad \text { и } \quad \int_{0}^{1} h^{\prime}(t) f(t) g(t) d t .
$$

Действительно, первый из них получается известным путем в случае двух переменных сомножителей, если таковыми брать $f(t)$ и $g(t) h(t)$; для получения же остальных двух интегралов берутся в качестве двух сомножителей $g(t)$ и $f(t) h(t)$, а также $h(t)$ н $f(t) g(t)$. Очевидно, что сумма этих трех интегралов равна всему приросту гіроизведения и выражается разностью $f(1) g(1) h(1)-f(0) g(0) h(0)$, так как

$$
\frac{d}{d t} f(t) g(t) h(t)=f^{\prime}(t) g(t) h(t)+f(t) g^{\prime}(t) h(t)+f(t) g(t) h^{\prime}(t) .
$$

Ход решения задачи остается в принципе тем же в случае любого числа переменных сомножителей и приводит к следующему результату:

если произведение $f(t) g(t) \ldots v(t)$ обозначить $P(t)$ и рассматривать его изменение в интервале $0 \leqslant t \leqslant 1$, причем $f(0)=1=g(0)=\ldots=v(0)$ и $P(1)=\Psi$, то его прирост $\Psi-1$ распадается на части

$$
\int_{0}^{1} f^{\prime}(t) \frac{P(t)}{f(t)} d t, \quad \int_{0}^{1} g^{\prime}(t) \frac{P(t)}{g(t)} d t, \ldots, \int_{0}^{1} v^{\prime}(t) \frac{P(t)}{v(t)} d t
$$

выражающне, соответственно, влияние изменения первого, второго, ... последнего сомножителей.

\section{4. Примеры и замечания}

1. Если линия $C F$ (фиг. 2) дана уравнениями $x=\lambda^{t}$ и $y=\mu^{t}$, то прирост произведения разделяется на части $\int_{0}^{1}\left(\lambda^{t} \ln \lambda\right) \mu^{t} d t$ и $\int_{0}^{1}\left(\mu^{t} \ln \mu\right) \lambda^{t} d t$, которые в случае $\lambda \mu \neq 1$ вычисляются так:

$$
\int_{0}^{1}\left(\lambda^{t} \ln \lambda\right) \mu^{t} d t=(\ln \lambda) \int_{0}^{1}(\lambda \mu)^{t} d t=\frac{\ln \lambda}{\ln (\lambda \mu)}(\lambda \mu-1)
$$




$$
\int_{0}^{1}\left(\mu^{t} \ln \mu\right) \lambda^{t} d t=(\ln \mu) \int_{0}^{1}(\lambda \mu)^{t} d t=\frac{\ln \mu}{\ln (\lambda \mu)}(\lambda \mu-1)
$$

а в случае $\lambda \mu=1$ :

$$
\begin{aligned}
& \int_{0}^{1}\left(\lambda^{t} \ln \lambda\right) \mu^{t} d t=(\ln \lambda) \int_{0}^{1} d t=\ln \lambda \\
& \int_{0}^{1}\left(\mu^{t} \ln u\right) \lambda^{t} d t=(\ln \mu) \int_{0}^{1} d t=\ln \mu .
\end{aligned}
$$

При любом числе сомножителей, зависящих от параметра $t$ экспоненциально, нх пронзведение само - экспоненциальная функция того же параметра, т. е. $\lambda^{t} \mu^{t} \ldots \omega^{t}=\Psi^{t}$, причем $\lambda \mu \ldots \omega=\Psi$. Ввиду того, что в случае $\Psi \neq 1$

$$
\int_{0}^{1}\left(\lambda^{t} \ln \lambda\right) \mu^{t} \ldots \omega^{t} d t=(\ln \lambda) \int_{0}^{1} \Psi^{t} d t=\frac{\ln \lambda}{\ln \Psi}(\Psi-1),
$$

а в случае $\Psi=1$ :

$$
\int_{0}^{1}\left(\lambda^{t} \ln \lambda\right) \mu^{t} \ldots \omega^{t} d t=(\ln \lambda) \int_{0}^{1} d t=\ln \lambda
$$

общий результат сводится к следующему правилу:

прнрост произведения величин, зависящих от одного и того же параметра экспоненциально, разделяется между ними (в соответствии с их влиянием на произведение) пропорционально логарифмам их коэффициентов изменения при условии, что прирост произведения не равен нулю; в случае же, когда прирост произведения нуль, влияние каждого сомножителя выражается натуральным логарифмом его коэффициента изменения (помноженным на значение произведения, если последнее не взято единицей).

Итак, экспоненциальная зависимость сомножителей от $t$ влечет за собой нормальное разделение прироста произведения.

2. Если линия $C F$ (фиг. 2) прямая $x=1+\alpha t, y=1+\beta t$, то прирост пронзведения разделяется на части

$$
\int_{0}^{1} \alpha(1+\beta t) t d \text { и } \int_{0}^{1} \beta(1+\alpha t) d t,
$$

значения которых просто $\alpha\left(1+\frac{\beta}{2}\right)$ и $\beta\left(1+\frac{a}{2}\right)$.

При трех переменных сомножителях, зависящих от $t$ линейно, их влияние на произведение выражается следующими частями его прироста:

$$
\begin{aligned}
& \int_{0}^{1} \alpha(1+\beta t)(1+\gamma t) d t, \quad \int_{0}^{1} \beta(1+\alpha t)(1+\gamma t) d t \quad \text { и } \quad \int_{0}^{1} \gamma(1+\alpha t)(1+\beta t) d t \text {, т. е. } \\
& \alpha\left(1+\frac{\beta+\gamma}{2}+\frac{\beta \gamma}{3}\right), \quad \beta\left(1+\frac{\alpha+\gamma}{2}+\frac{a \gamma}{3}\right) \quad \text { и } \quad \gamma\left(1+\frac{a+\beta}{2}+\frac{a \beta}{3}\right) .
\end{aligned}
$$


3. Некоторые трудности могут возникнуть при толковании того факта, что задача разделения прироста произведения имеет единственное и вполне определенное решение, если все переменные сомножители даны в виде функций одного и того же параметра, и что задача неопределенна, если ход изменения одного или нескольких сомножителей неизвестен.

Напрашивается, например, следующий «вывод». Знание хода изменения всех переменных сомножителей дает возможность считать один из них независимым переменным и все остальные его функциями, так что произведение изменяется, собственно, под влиянием одного этого сомножителя, поскольку все остальные сомножнтели зависят от него и переносят часть его влияния на произведение, но своего собственного влияния оказывать не могут. Весь прирост произведения обязан тогда нзменению этого единственного незавнсимого сомножителя, и задача разделения прироста явно бессмысленна. А полный смысл она имеет только в случае, когда у сомножителей нет никакой взаимосвязи, но тогда она, к сожалению, совсем неопеделенна.

В чем заключается оплошность, допускаемая при таком выводе? Пусть $u$ и $v$ изменяются во времени $t$ и даны как известные функции этого параметра $t$ :

$$
u=f(t) \quad \text { и } \quad v=g(t) \quad \text { при } 0 \leqslant t \leqslant 1 .
$$

Хотя все обстоятельства, причастные к изменению величины $u$, могут быть в какой угодно мере независимыми от тех, которые определяют ход изменения величины $v$, уже один тот факт, что $u$ и $v$ известны как функции времени, позволяет путем элиминации времени из системы уравнений $\left\{\begin{array}{l}u=f(t) \\ v=g(t)\end{array}\right.$ получить одно уравнение с двумя переменными $u$ и $v$; если, например, из $u=f(t)$ следует $t=\varphi(u)$, то $v=g(\varphi(u))$.

В каком смысле это уравнение означает, что $v$ зависит от $u$ ? Қонечно, не в том смысле, что при любых обстоятельствах и в любое время, как только величине $u$ будет в действительности дано какое-нибудь значение $a$, в силу этого уравнения величина $v$ будет непременно иметь значение $g(\varphi(a))$. Путем элиминации времени установлено прямое соответствие между значениями величин $u$ и $v$, но это соответствие не означает ничего большего, чем одновременность этих значений в рассматриваемом явлении (которое иногда может иметь характер неповторимого сложного события). Элиминация времени из системы уравнений не превращает явление в безвременный процесс (да и условие $0 \leqslant t \leqslant 1$ не отменяется) и вообще не вызывает ничего небывалого в природе. Ни до ни после элиминации нельзя полагать, чтобы подбор значений величины $u$ мог оказывать влияние на течение времени - согласно уравнению $t=\varphi(u)$, а таким путем и на величину $v$. Следовательно, $v=g(\varphi(u))$ означает зависимость величины $v$ от $u$ в крайне ограниченном смысле, указывая только, какие значения величин $u$ и $v$ одновременны в данном индивидуальном процессе их изменения.

4. Если известны значения всех сомножителей только до и после изменений, то задача разделения прироста произведения неопеделенна. Часто бывает, что некоторые сомножители не принимают никаких значений в промежуточные времена. Например, если один сомножитель означает посевную площадь сельскохозяйственного предприятия в рассматриваемом году и другой сомножитель - среднюю урожайность полей, то их произведение выражает весь урожай, 'и можно установить его прирост по сравнению с урожаем прошлого года, но нельзя говорить об изменениях урожайности полей в течение года, например, зимой или весной.

Неопределенные задачи принято решать так, что при однотипных задачах решение опирается на одни и те же предположения, возможно более простые и реальные. Одним нз наиболее естественных предположений о ходе изменения величин следует считать предпосылку, что они зависят от времени экспоненциально, изменяются по закону еорганического роста». Такому предположению соответствует нормальное разделение прнроста произведения между переменными сомножителями.

Поступила в редакцию

19. XII 1961 


\section{KORRUTISE KASVU JAOTAMINE}

\section{A. Humal,}

\section{ENSV Teaduste Akadeemia akadeemik}

Resümee

Kui kahe suuruse korrutises nad mõlemad muutuvad, siis võib küsida, missugune osa korrutise kasvust vastab ühe suuruse mōjule ja missugune teise mõjule. Olgu suuruste algväärtused $p, q$ ja lōppväärtused $\lambda p, \mu q$. Korrutisel $p q$ on siis kasv $(\lambda \mu-1) p q$ ning üsna elementaarsete kaalutlustega jōutakse tulemusele, et selle jaotamine osadeks

$\frac{\log \lambda}{\log \lambda+\log \mu}(\lambda \mu-1) p q$ ja $\frac{\log \mu}{\log \lambda+\log \mu}(\lambda \mu-1) p q$ täidab teatavaid asjakohaseid tingimusi; ta nimetatakse korrutise kasvu normaaljaotuseks.

Kui kōigi tegurite muutumine on oma käigu poolest teada, siis osutub korrutise kasvu jagunemine (üksikute tegurite môju järgi) üheselt arvutatavaks. Olgu tegurid antud ühe parameetri $t$ funktsioonidena $f(t), g(t), \ldots, v(t)$ (kusjuures $0 \leqslant t \leqslant 1$ ). Kui mōõtühikud nii valitakse, et $f(0)=1=g(0)=\ldots=v(0)$, ja tähistatakse $f(1)=\lambda, g(1)=\mu, \ldots, v(1)=\omega$, $\lambda \mu \ldots \omega=\Psi$ ning $f(t) g(t) \ldots v(t)=P(t)$, siis jaguneb korrutise kasv $\Psi-1$ osadeks

$$
\int_{0}^{1} f^{\prime}(t) \frac{P(t)}{f(t)} d t, \quad \int_{0}^{1} g^{\prime}(t) \frac{P(t)}{g(t)} d t, \ldots, \quad \int_{0}^{1} v^{\prime}(t) \frac{P(t)}{v(t)} d t,
$$

mis vastavad tegureile $f(t), g(t), \ldots, v(t)$. Juhul, kui teguriteks on eksponentiunktsioonid $\lambda t, \quad \mu^{t}, \ldots, \omega^{t}$, saadakse vastavate osadena $\frac{\ln \lambda}{\ln \Psi}(\Psi-1), \frac{\ln \mu}{\ln \Psi}(\Psi-1), \ldots, \frac{\ln \omega}{\ln \Psi}(\Psi-1)$, seega normaaljaotus.

Saabus toimetusse

19. XII 1961

\section{DIE TEILUNG DES PRODUKTZUWACHSES}

\section{A. Humal,}

\section{Mitglied der Akademie der Wissenschaften der Estnischen SSR}

\section{Zusammenfassung}

Wenn im Produkte zweier Grössen die beiden sich verändern, kann man fragen, welcher Teil seines Zuwachses dem Einfluss der einen Grösse entspricht und welcher dem Einfluss der anderen. Die Anfangswerte der Grössen seien $p, q$ und die Endwerte $\lambda p, \mu q$. Das Produkt hat dann den Zuwachs $(\lambda \mu-1) p q$, und durch ganz elementare Erwägungen gelangt man zum Ergebnis, dass seine Teilung in $\frac{\log \lambda}{\log \lambda+\log \mu}(\lambda \mu-1) p q$ und $\log \mu$ $\overline{\log \lambda+\log \mu}(\lambda \mu-1) p q$ gewisse sachgemässe Bedingungen erfüllt; sie wird die Normalteilung des Produktzuwachses genannt.

Ist die Veränderung aller Faktoren in ihrem Verlauf völlig bekannt, so erweist sich die Zerteilung des Produktzuwachses (gemäss dem Einfluss einzelner Faktoren) eindeutig berechenbar. Die Faktoren seien als Funktionen $f(t), g(t), \ldots, v(t)$ eines Parameters $t$ gegeben $(0 \leqslant t \leqslant 1)$. Wählt man die Masseinheiten so, dass $f(0)=1=g(0)=\ldots=v(0)$, und bezeichnet man $f(1)=\lambda, g(1)=\mu, \ldots, v(1)=\omega, \lambda \mu \ldots \omega=\Psi$ und $f(t) g(t) \ldots v(t)=P(t)$, so zerfällt der Produktzuwachs $\Psi-1$ in Teile

$$
\int_{0}^{1} f^{\prime}(\mathrm{t}) \frac{P(t)}{f(t)} d t, \quad \int_{0}^{1} g^{\prime}(t) \frac{P(t)}{g(t)} d t, \ldots, \quad \int_{0}^{1} v^{\prime}(t) \frac{P(t)}{v(t)} d t,
$$

die den Faktoren $f(t), g(t), \ldots, v(t)$ entsprechen. Im Falle, dass die Faktoren Exponentialfunktionen $\lambda^{t}, \mu^{t}, \ldots, \omega^{t}$ sind, hat man die entsprechenden Teile $\frac{\ln \lambda}{\ln \Psi}(\Psi-1), \frac{\ln \mu}{\ln \Psi}(\Psi-1), \ldots, \frac{\ln \omega}{\ln \Psi}(\Psi-1)$, also die Normalteilung. 